Editorial comment

\title{
The interference of pain with task performance: Increasing ecological validity in research
}

Dimitri M. L. Van Ryckeghem ${ }^{1,2, *}$

Affiliation(s):

${ }^{1}$ Institute for Health and Behaviour, INSIDE, University of Luxembourg, Luxembourg

${ }^{2}$ Department of Experimental Clinical and Health Psychology, Ghent University, Belgium

*Corresponding author

Dimitri M. L. Van Ryckeghem

Affiliation address:

Dimitri Van Ryckeghem

Université du Luxembourg

Maison des Sciences Humaines, Porte des Sciences 11, L-4366 Esch-sur-Alzette

Telephone: (+352) 4666449241

Fax: /

Email: Dimitri.VanRyckeghem@uni.lu

Aknowledgements

Dimitri Van Ryckeghem is supported by the European Union's Horizon 2020 research and innovation programme under the Marie Sklodowska-Curie grant No 706475. 
In this issue of the Scandinavian Journal of Pain you will find a paper by David Moore and Anna Law, entitled "The disruptive effects of pain on multitasking in a virtual errands task." in which the authors investigate the impact of pain upon complex task performance [1].

\section{Task interference by pain depends upon pain, task characteristics and individual differences}

Research investigating the impact of pain upon task performance has gained increasing interest during the last decades. Most often, research findings have shown that pain draws attention and interferes with task performance and goals people pursue in daily life [2,3]. Importantly, this research has indicated that the magnitude of task interference by pain is dependent upon a multitude of factors. A first set of factors relates to the characteristics of the pain. Indeed, earlier research has shown that interference by pain is larger in case the pain is novel (e .g., [4]), more intense (e. g., [5]), unpredictable (e. g., [6]) and has a large threat value (e. g., [7]). A second set of factors relates to individual differences of people that experience pain. For example, Van Damme and colleagues indicated that pain catastrophizing may increase the impact of pain upon task performance [8]. A last array of factors relates to the particular characteristics of a task people are performing. For example, Moore and colleagues (2012) suggested that tasks requiring higherorder processes are more susceptible for interference by pain [9], whereas Verhoeven and colleagues (2010) found that increasing task importance can reduce task interference by pain [10].

\section{Increasing ecological validity of experimental designs}

The study of Moore and Law addresses the last two domains, i.e., individual differences and task characteristics that influence the impact of pain upon task performance. By using the Edinburgh Virtual Errands Task (EVET), Moore and Law further earlier findings by investigating the impact of pain upon 'virtual' daily life tasks. In doing so, the current study attempts to provide an answer to the call for an increase of ecological validity in researching task interference by pain (e.g., [11]). Indeed, until now, most research has investigated the effect of acute or chronic pain upon standard experimental tasks. Although those study designs enable to research the effect of pain upon specific cognitive functions (e.g., working memory), researching the presence of pain when performing more ecological valid (complex) 'virtual' tasks may prove helpful to investigate the impact of pain upon complex daily activities people usually engage in.

\section{Limited impact of pain upon task performance: the role of cognitive intrusion}

Results of the current study may be surprising as, in contrast with expectations, no overall detriment in task performance was found due to the presence of pain. Only in the group of individuals who reported larger cognitive intrusion by pain in general (as measured with the Experience of Cognitive Intrusion of Pain scale), it was found that pain resulted in more violation of task rules (e. g., by entering incorrect rooms). 
However, several issues may explain the (lack of) findings in the current study of Moore and Law. First, the authors opted to use only low intense pain stimuli, which may have decreased the impact of pain upon task performance. Furthermore, the EVET task is of a short duration ( 8 minutes). It may well be that people are able to protect their task performance from interruption by pain by increasing the levels of effort invested in the task as they anticipate that pain may decrease their task performance [5]. Increasing pain intensity and task duration could accordingly have impacted upon the level of task interference. However, these explanations are speculative and need further investigation.

\section{New methodologies to unravel the influence of pain and task characteristics}

It is in particular future research possibilities to answer above-mentioned and other theory-driven questions in the context of more ecological valid (complex) 'virtual' tasks that make current paradigm innovative. The use of the EVET (or similar paradigms) may help to unravel the influence of pain and task characteristics upon the extent to which pain interferes with task performance. Current paradigm easily allows to manipulate the characteristics of the virtual daily tasks. For example, one may increase the importance or motivational relevance of some tasks while decreasing the importance or motivational relevance for other tasks. Similarly, one may manipulate the pain or threat value related to a particular task. Manipulating task characteristics of these more ecological valid (complex) 'virtual' tasks that aim to mimic daily life situations may provide important insights into the impact of pain upon complex daily activities.

\section{Some further steps to increase ecological validity in the investigation of task interference by pain}

Further steps to increase ecological validity in investigating task interference by pain are ahead of us and may take into account the findings and problems of the current study. First, researchers may use naturally-occurring pain (e. g., headache; [12]) rather than experimentally induced pain when performing virtual daily tasks. Furthermore, research may also make use of advances in the virtual reality to mimic real world tasks even better. By using virtual reality, people can be requested to actually perform daily tasks (e.g., bending over, reaching for a paper, walking around) in a virtual world. Using this approach people experiencing pain (e.g., chronic low back pain) could be asked to engage in ecological valid (complex) tasks that are perceived threatening and painful, while manipulating the task characteristics (e.g., task importance). In sum, each of these advancements to investigate the impact of pain upon task execution may give us increasing insights in the conditions under which pain interferes with daily tasks and how we could eventually reduce its impact. 


\section{References}

[1] Moore DJ, Law AS. The disruptive effects of pain on multitasking in a virtual errands task. Scand J Pain 2017;16: in current issue.

[2] Eccleston C, Crombez G. Pain demands attention: A cognitive-affective model of the interruptive function of pain. Psychol Bull 1999;125:356-366.

[3] Vlaeyen JWS, Morley S, Crombez G. The experimental analysis of the interruptive, interfering, and identity-distorting effects of chronic pain. Behav Res Ther 2016;86:23-34.

[4] Legrain V, Bruyer R, Guérit JM, Plaghki L. Nociceptive processing in the human brain of infrequent task-relevant and task-irrelevant noxious stimuli. A study with event-related potentials evoked by CO2 laser radiant heat stimuli. Pain 2003;103:237-248.

[5] Van Ryckeghem DML, Crombez G, Eccleston C, Liefooghe B, Van Damme S. The interruptive effect of pain in a multitask environment: an experimental investigation. J Pain 2012;13:131-138

[6] Crombez G, Baeyens F, Eelen P. Sensory and temporal information about impeding pain - the influence of predictability on pain. Behav Res Ther 1994;32:611-622.

[7] Van Damme S, Crombez G, Van Nieuwenborgh-De wever K, Goubert L. Is distraction less effective when pain is threatening? An experimental investigation with the cold pressor task. Eur J Pain 2008;12:60-67.

[8] Van Damme S, Crombez G, Eccleston C. Disengagement from pain: the role of catastrophic thinking about pain. Pain 2004;107:70-76.

[9] Moore DJ, Keogh E, Eccleston C. The interruptive effect of pain on attention. Q J Exper Psychol 2012;65:565-586.

[10] Verhoeven K, Crombez G, Eccleston C, Van Ryckeghem DML, Morley S, Van Damme S. The role of motivation in distracting attention away from pain: an experimental study. Pain 2010;149:229234.

[11] Keogh E, Moore DJ, Duggan GB, Payne SJ, Eccleston C. The disruptive effects of pain on complex cognitive performance and executive control. PLoS ONE 2013;8:e83272

[12] Attridge N, Eccleston C, Noonan D, Wainwright E, Keogh E. Headache Impairs Attentional Performance: A Conceptual Replication and Extension. J Pain 2017;18:29-41 